\title{
Perfil algométrico e funcional de pacientes com dor lombar atendidos em um ambulatório de fisioterapia como parte de um projeto de desenvolvimento de recurso terapêutico
}

\author{
Algometric and functional profile of patients with low back pain treated at a physiotherapy \\ outpatient clinic as part of a therapeutic resource development project
}

Perfil algométrico y funcional de pacientes con mosquio lumbar tratados en un ambulatorio de fisioterapia como parte de un proyecto de desarrollo de recursos terapéuticos

Júlio Cesar da Rocha Alves ${ }^{1}$, Paula Luiza Matni dos Santos ${ }^{1}$, Carla Daniela Santiago Oliveira ${ }^{1}$, Laís Socorro Barros da Silva ${ }^{1}$, Ana Paula Ricardo Cavalcanti ${ }^{1}$, Ediléa Monteiro de Oliveira $^{1}$, Luiz Fábio Magno Falcão ${ }^{1 *}$.

\section{RESUMO}

Objetivo: Delinear o perfil algométrico e funcional de pacientes com dor lombar como parte de um projeto de recurso terapêutico. Métodos: Trata-se de um estudo transversal descritivo de caráter quantitativo, no qual participaram 40 indivíduos diagnosticados com dor lombar. Foi realizada uma avaliação da dor por meio da Escala Visual Analógica e algometria digital, da flexibilidade do tronco por meio do teste de Sentar e Alcançar, e do nível de incapacidade pelo Questionário Roland-Morris. Resultados: Entre os 40 participantes avaliados 15 são homens e 25 mulheres, com idade média de 46,67 anos. A média total registrada pela Escala Visual Analógica foi de 3,7 e na avaliação algométrica digital a média total foi de 3,64 $\mathrm{Kg} / \mathrm{cm}^{2}$. Na avaliação de incapacidade a pontuação média foi de $12,35 \mathrm{com}$ alta correlação positiva com os níveis de dor, e alta correlação negativa com a flexibilidade do tronco. Conclusão: Foi possível observar resultados potencialmente úteis ao estudo dos sintomas e repercussões funcionais da dor lombar, e no aprimoramento dos meios de tratamento desta condição, embasando a definição de requisitos no desenvolvimento de um recurso terapêutico.

Palavras-chave: Dor lombar, Fisioterapia, Ortopedia.

\begin{abstract}
Objective: To delineate the algometric and functional profile of patients with low back pain as part of a therapeutic resource project. Methods: This is a descriptive cross-sectional study of quantitative character, in which 40 individuals diagnosed with low back pain participated. Pain was evaluated using the Visual Analog Scale and digital algometry, trunk flexibility through the Sit and Reach test, and the level of disability by the Roland-Morris Questionnaire. Results: Among the 40 participants evaluated, 15 were men and 25 women, with a mean age of 46.67 years. The total mean recorded by the Visual Analog Scale was 3.7 and in the digital algometric evaluation the total mean was $3.64 \mathrm{Kg} / \mathrm{cm}^{2}$. In the disability assessment, the mean score was 12.35 with a high positive correlation with pain levels, and a high negative correlation with trunk flexibility. Conclusion: It was possible to observe potentially useful results for the study of symptoms and functional repercussions of low back pain, and on the improvement of the means of treatment of this condition, basing the definition of requirements in the development of a therapeutic resource.
\end{abstract}

Keywords: Low back pain, Physical therapy specialty, Orthopedics.

RESUMEN

Objetivo: Delinear el perfil algométrico y funcional de pacientes con mosquiso lumbar como parte de un proyecto de recursos terapéuticos. Métodos: Se trata de un estudio descriptivo transversal de carácter cuantitativo, en el que participaron 40 individuos diagnosticados de abajo. El dolor se evaluó mediante la

1 Universidade do Estado do Pará (UEPA), Belém - PA. *E-mail: fabiofalcao29@yahoo.com.br

SUBMETIDO EM: 10/2021

ACEITO EM: 10/2021

PUBLICADO EM: 10/2021 
Escala Analógica Visual y la algometría digital, la flexibilidad del tronco a través de la prueba Sit and Reach, y el nivel de discapacidad mediante el Cuestionario Roland-Morris. Resultados: Entre los 40 participantes evaluados, 15 eran hombres y 25 mujeres, con una edad media de 46,67 años. La media total registrada por la Escala Analógica Visual fue de 3,7 y en la evaluación algométrica digital la media total fue de 3,64 $\mathrm{Kg} / \mathrm{cm}^{2}$. En la evaluación de la discapacidad, la puntuación media fue de 12,35 con una alta correlación positiva con los niveles de dolor y una alta correlación negativa con la flexibilidad del tronco. Conclusión: Fue posible observar resultados potencialmente útiles para el estudio de los sintomas y las repercusiones funcionales del dolor lumbar, y en la mejora de los medios de tratamiento de esta afección, basando la definición de requerimientos en el desarrollo de un recurso terapéutico.

Palabras clave: Dolor de la región lumbar, Fisioterapia, Ortopedia.

\section{INTRODUÇÃO}

A coluna lombar é uma complexa estrutura anatômica, cuja função biomecânica principal é suportar cargas e fornecer proteção para o tecido mole neural. A dor lombar ocorre entre as margens inferiores das costelas e as dobras das nádegas e é comumente acompanhada de dor em uma ou ambas as pernas. (HARTVIGSEN J, et al., 2018; NIM CG, et al., 2021).

Entre as características clínicas presentes em pacientes com dor lombar, encontram-se alterações funcionais, como instabilidade estática ou dinâmica na coluna, reduções na amplitude de movimento, e distúrbios somatossensoriais. De acordo com a National Health and Medical Research Council a repercussão mais comum da dor lombar é a diminuição dos movimentos da coluna vertebral (CHETTY L, 2017; GRANDE-ALONSO M, et al., 2020).

A dor lombar é a condição musculoesquelética mais comum que afeta a qualidade de vida, especialmente se persistente. Esta condição tem sido descrita na literatura como um dos agravos de saúde que mais levam a desfechos negativos de incapacidade física no mundo, ocasionando desgaste econômico e social para os sistemas de saúde e para os próprios pacientes que experimentam o sintoma (ZAINA F, et al., 2020; HARTVIGSEN J, et al., 2018).

A incidência da dor lombar tende a aumentar com a idade, sendo mais prevalente entre adultos na faixa etária de 40 aos 69 anos. Apresenta-se como uma das principais causas de incapacidade física no mundo e a responsável pela maior quantidade de anos vividos com incapacidade e redução da capacidade para 0 trabalho dentre todas as outras condições de saúde (POLLI GR, et al., 2018).

Segundo dados do DATASUS de 2013 a 2018, foram notificados 59.954 casos de dor lombar no sistema público de saúde no Brasil, resultando em uma prevalência média de 4,87 por 100.000 pessoas no Brasil por ano. Observou-se uma tendência crescente nos casos de dor lombar ao longo dos anos, de 9.360 casos em 2013 para 10.227 casos em 2018 (MENDONÇA AG, 2020).

Paralelamente à exploração dos aspectos mecânicos envolvidos na dor lombar, pesquisas também têm explorado os mecanismos subjacentes da própria experiência dolorosa. Isso inclui testes sensoriais quantitativos que possam quantificar a percepção individual da dor em resposta a estímulos nociceptivos controlados (GRAVEN-NIELSEN T e ARENDT-NIELSEN L, 2010).

O tratamento da dor lombar visa principalmente o alívio da dor, podendo ser farmacológico, cirúrgico, não invasivo e não farmacológico, dentre os quais a fisioterapia se destaca podendo levar à redução da dor e da tensão muscular. Existem diversos recursos fisioterapêuticos como exercícios terapêuticos, mobilização neural e tração da coluna vertebral, dentre outros (RAMOS M, et al., 2020).

A motivação do estudo consiste na da importância de investigar os mecanismos envolvidos na lombalgia e suas repercussões na qualidade de vida, a fim de embasar novos recursos de tratamento para esta condição. Objetiva-se, portanto, delinear o perfil algométrico e funcional de pacientes ambulatoriais com dor lombar, estabelecendo possíveis associações entre as variáveis. Espera-se que os resultados auxiliem na determinação de necessidades dos usuários e na definição de requisitos de projeto no desenvolvimento de um recurso terapêutico voltado a esta clientela. 


\section{MÉTODOS}

Trata-se de um estudo transversal descritivo, de caráter quantitativo, baseado na determinação e análise do perfil álgico e funcional de pacientes com queixa de dor lombar. O estudo foi desenvolvido como parte do projeto "Desenvolvimento de um dispositivo posicional voltado a pacientes com redução da mobilidade e da capacidade funcional devido à lombalgia" financiado por uma fundação de fomento à pesquisa na região amazônica. CAAE do projeto principal: 86112218.6.0000.5174.

O projeto foi submetido ao Comitê de Ética em Pesquisa (CEP) envolvendo seres humanos de uma universidade do estado do Pará e aprovado pelo parecer de № 2.702.012, conforme a Resolução do Conselho Nacional de Saúde № 466/2012. Os procedimentos do estudo foram executados respeitando as normas sanitárias relacionadas à pandemia da COVID-19.

Foram incluídos no estudo 40 participantes adultos, na faixa etária de 18 a 65 anos, de ambos os sexos, com diagnóstico de dor lombar confirmado por laudo médico, encaminhados à uma unidade de ensino e assistência em fisioterapia e terapia ocupacional de uma universidade pública no estado do Pará, pela rede de atendimento do Sistema Único de Saúde (SUS). Assim como nos estudos de Choi J, et al. (2015) e Carvalho AS (2015), foram excluídos participantes com histórico de cirurgia vertebral, tumores espinhais, infecções no disco intervertebral, doenças inflamatórias, fraturas vertebrais, osteoporose grave e processos anquilosantes da coluna

Os procedimentos de coleta de dados consistiram em uma avaliação composta por itens de identificação pessoal como idade, escolaridade, ocupação, avaliação da percepção álgica por meio da Escala Visual Analógica (EVA) e algometria digital, avaliação da flexibilidade do tronco por meio do Teste de Sentar e Alcançar (TSA), e do nível de incapacidade funcional pelo Questionário Roland-Morris (QRM).

Segundo Martinez JE, et al. (2011), a EVA é um instrumento unidimensional para a avaliação da intensidade da dor. Trata-se de uma linha com as extremidades numeradas de 0-10. Em uma extremidade da linha é marcada "sem dor" (0) e na outra "pior dor possível" (10). Para o estudo foi utilizada uma versão modificada com inclusão de ícones de faces, relacionados aos níveis de dor. A escala linear será representada com cores variando do verde (ausência ou níveis mínimos de dor) ao vermelho (maiores níveis de dor).

A algometria digital é uma técnica utilizada para quantificar a intensidade da dor e para localizar pontos dolorosos meio de estímulos de pressão, sendo esta diretamente relacionada à força aplicada e inversamente à área estimulada elevada ao quadrado, sendo os resultados expressos $\mathrm{em} \mathrm{Kg} / \mathrm{cm}^{2}$, ressaltando que níveis menores de leitura correspondem a maior sensibilidade álgica (BERNARDINO SN, 2012; SIVIERO D, et al., 2013). Utilizou-se no estudo o algômetro de pressão digital MEDDOR+ que possui uma carga máxima de $50 \mathrm{Kg}$, visor de LCD e medição digital microprocessada.

Para a medição, o examinador posicionou-se atrás do paciente, ambos de pé, em seguida, a ponteira do instrumento foi colocada em contato com a região paravertebral lombar do paciente, lateralmente a $2 \mathrm{~cm}$ do processo espinhoso L4. O examinador pressiona lentamente com a ponteira sobre o local, e o paciente orientado a reportar o início da dor à pressão, sendo o valor de força aplicada registrada pelo instrumento. A medição foi realizada bilateralmente, sendo calculada e registrada a média entre os dois registros (algometria média). É importante ressaltar que a leitura da algometria se refere à pressão necessária ao início da queixa álgica, ou seja, quanto menor o valor registrado, maior a sensibilidade álgica no local.

O TSA pode ser utilizado como guia para quantificação da flexibilidade do tronco e da cadeia muscular posterior (MARTINS ABQP, et al., 2016). Para o estudo foi utilizado como auxílio o instrumento Banco de Wells. O procedimento consiste em solicitar que, a partir da posição sentada e com os joelhos estendidos, 0 participante flexione o tronco para frente com a intenção de alcançar a máxima distância possível com os dedos no medidor, sem que seja necessário sentir dor para chegar ao seu limite. O movimento é realizado três vezes consecutivas, sendo considerada a medida de maior valor em centímetros.

O Questionário Roland-Morris (QRM) foi desenvolvido em 1983 por Roland e Morris com o objetivo de avaliar a incapacidade funcional de indivíduos com lombalgia. O questionário é constituído por 24 perguntas 
de autorresposta, que podem ser respondidas em até cinco minutos (MONTEIRO J, et al., 2010). As respostas consistem em "sim ou não" e são relacionadas às funções físicas com o intuito de avaliar a incapacidade funcional e o quanto a lombalgia afeta o indivíduo (SCHIMIDT GJ, et al., 2019).

O resultado corresponde à soma das respostas "sim" sendo atribuído 1 ponto para cada pergunta, a pontuação final pode variar de 0 no qual representa um indivíduo sem incapacidade e 24 indivíduos com incapacidade grave (MONTEIRO J, et al., 2010). Apesar do QRM originalmente não prever estratificações ou classes de incapacidade, foi utilizada no presente estudo uma estratificação em 6 grupos por níveis de incapacidade: leve (1 a 6), moderada (7 a 12), grave (13 a 18), e muito grave (19 a 24).

A análise dos dados foi feita por meio de estatística descritiva no software BioEstat 5.0 expressos por meio de percentuais, médias e desvios-padrão. Para a inferência estatística utilizou-se o teste do quiquadrado para comparar classes e a normalidade dos dados pelo teste Kolmogorov-Smirnov. Utilizou-se o Coeficiente de Correlação de Pearson ( $r$ ) para determinação de correlação entre variáveis. O Coeficiente de Pearson que pode variar de $-1 \mathrm{a}+1$, e quanto mais próximos desses valores, mais forte a associação das variáveis, sendo que $o$ escore zero desse coeficiente indica ausência de correlação. $O$ valor $p$ de significância foi definido em 0,05 .

\section{RESULTADOS}

Foram submetidos aos procedimentos de avalição 40 pacientes, sendo 15 homens e 25 mulheres, com idade média de $46,67( \pm 12,36)$ anos. A ocupação mais frequente entre os participantes foi a de "serviços domésticos" referida em 12 casos (30\%). O nível de escolaridade predominante foi o "ensino médio completo" com 18 ocorrências (45\%). Em relação ao diagnóstico clínico, o mais prevalente foi a "lombalgia" ocorrendo em 19 casos (47,5\%). A queixa principal predominante foi a "dor" sendo referida por 34 participantes (85\%), sendo esta considerada "crônica" em 21 casos $(77,5 \%)$.

Em relação ao perfil álgico, na avaliação analógica a média total registrada pela EVA foi de $3,7( \pm 2,11)$, sendo que na distribuição entre os grupos de níveis de dor, o nível mais frequente foi o de "dor moderada" com 15 ocorrências relatadas, representando $37,5 \%$ (Figura 1). Na avaliação algométrica digital, a média total foi de $3,64 \mathrm{Kg} / \mathrm{cm}^{2}( \pm 2,08)$. Na distribuição das médias algométricas digitais por grupos de níveis da EVA, as menores leituras $\left(2.10 \mathrm{Kg} / \mathrm{cm}^{2}\right)$ foram registradas nos grupos de dor "severa" da EVA. O cálculo de correlação entre os níveis de EVA média e as médias de algometria digital resultou em um Coeficiente de Pearson de $-0,35(p=0,50)$ indicando baixa correlação negativa e sem significância estatística (Tabela 1).

Figura 1 - Número de participantes do estudo por grupos de níveis de dor avaliado pela EVA.

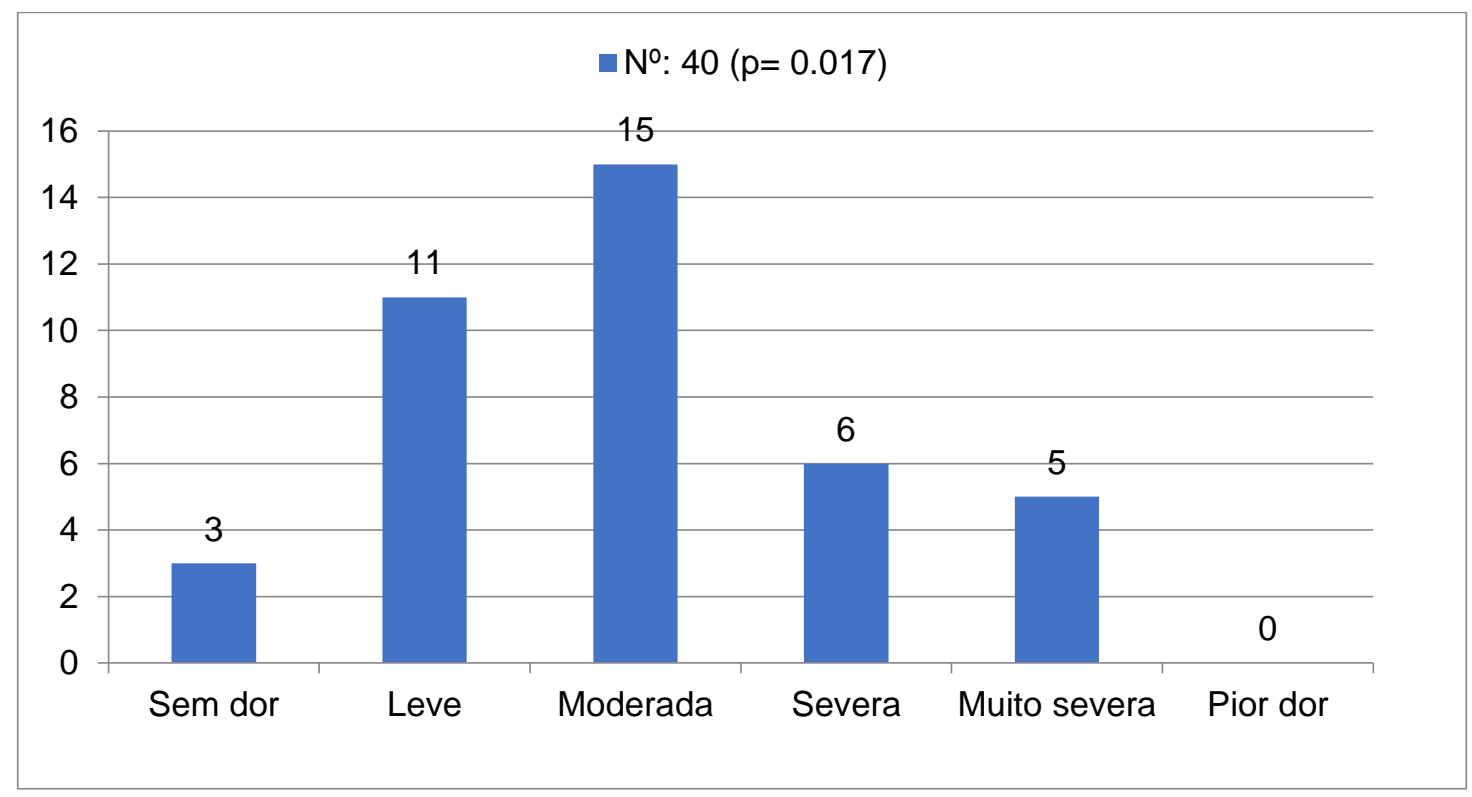

Fonte: Alves JCR, et al., 2021. 
Tabela 1 - Valores médios de algometria digital distribuídos por níveis de dor da EVA, n=40.

\begin{tabular}{|c|c|c|c|c|c|c|}
\hline Níveis de dor (EVA) & № & $\%$ & EVA Média & $\mathrm{Dp}^{1}$ & Alg. Média & $\mathrm{Dp}^{2}$ \\
\hline Sem dor & 3 & 7.5 & 0 & 0 & 4.63 & 2.22 \\
\hline Leve & 11 & 27.5 & 1.91 & 0.30 & 3.82 & 1.88 \\
\hline Moderada & 15 & 37.5 & 3.8 & 0.41 & 3.72 & 1.85 \\
\hline Severa & 6 & 15 & 5.33 & 1.85 & 2.10 & 1.85 \\
\hline Muito severa & 5 & 12.5 & 7.6 & 3.16 & 4.23 & 3.16 \\
\hline \multirow{2}{*}{ Total } & 40 & 100 & 3.72 & 2.94 & 3,7 & 0.96 \\
\hline & $p=0.017$ & & \multicolumn{4}{|c|}{$r=-0.35(p=0.50)$} \\
\hline
\end{tabular}

Legenda: №: Número de participantes. EVA Média: Valor médio de dor referida. Alg. Média: Valor médio da algometria digital em $\mathrm{Kg} / \mathrm{cm}^{2}$. $\mathrm{Dp}^{1}$ : Desvio-padrão referente à EVA Média. Dp²: Desvio-padrão referente à Alg. Média. r: Coeficiente de Correlação de Pearson. Fonte: Alves JCR, et al., 2021.

No tocante aos achados funcionais do estudo, obteve-se na avaliação do nível de incapacidade pelo QRM a pontuação média de 12,35 ( $\pm 5,20)$. Na distribuição entre as estratificações de níveis de incapacidade, observou-se o nível de "incapacidade grave" como o mais prevalente, verificado em 15 casos entre os avaliados representando $37,5 \%$ (Figura 2).

$\mathrm{Na}$ apresentação dos valores médios de pontuação pelo QRM, distribuídos por níveis de dor relatados na EVA, verificou-se a associação do nível de dor muito severa com a média de pontuação mais elevada do QRM (16 pontos). O cálculo de correlação entre os níveis de dor da EVA e o QRM médio resultou em um Coeficiente de Pearson de 0,91 ( $\mathrm{p}=0,03)$, indicando correlação positiva com significância estatística (Tabela 2).

Figura 2 - Distribuição dos participantes entre os de níveis de incapacidade medidos pelo QRM

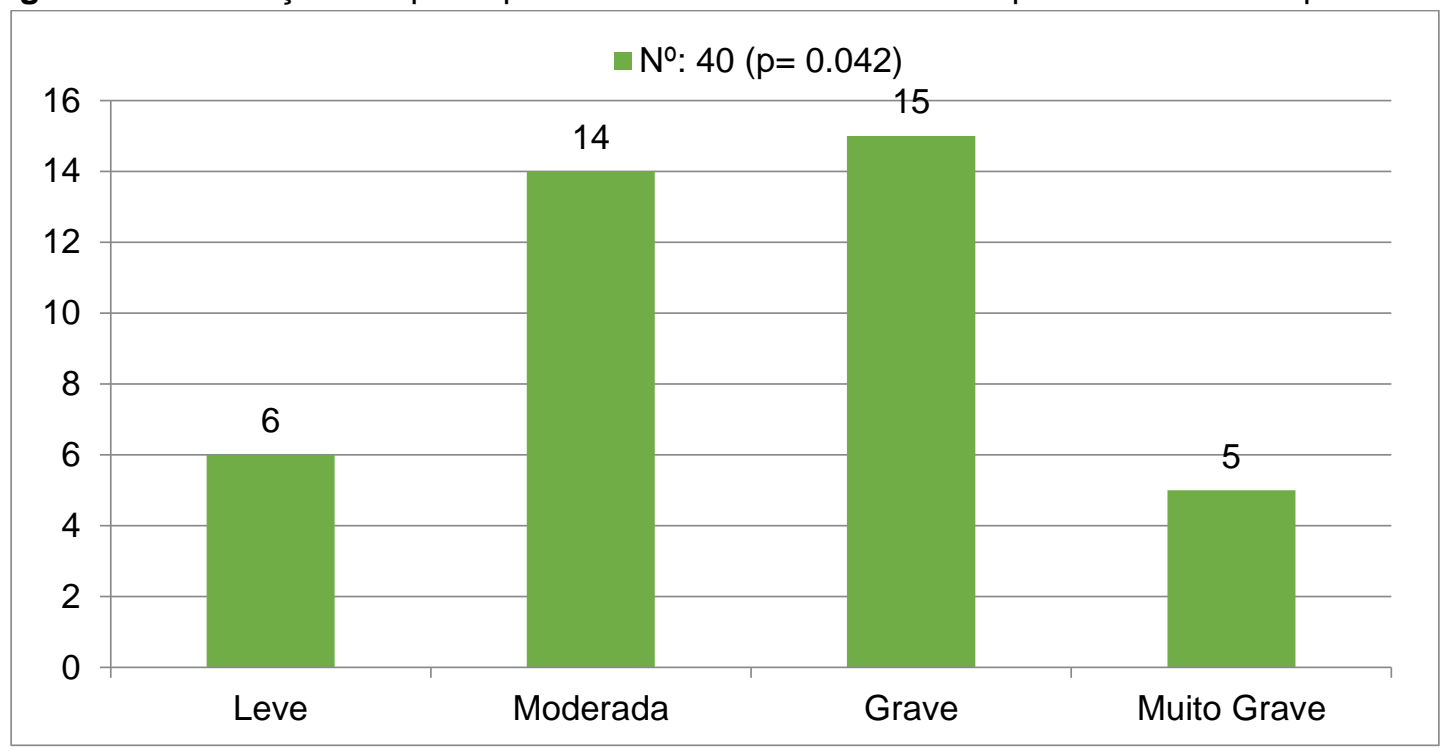

Fonte: Alves JCR, et al., 2021.

Tabela 2- valores médios de pontuação no QRM distribuídos por níveis de dor relatados na EVA, $n=40$.

\begin{tabular}{|c|c|c|c|c|c|c|}
\hline Níveis de dor EVA & № & $\%$ & EVA Média & $D p^{1}$ & QRM Médio & $D p^{2}$ \\
\hline Sem dor & 3 & 7.5 & 0 & 0 & 6.67 & 3.79 \\
\hline Leve & 11 & 27.5 & 1.91 & 0.30 & 12 & 4.98 \\
\hline Moderada & 15 & 37.5 & 3.8 & 0.41 & 12.2 & 5.91 \\
\hline Severa & 6 & 15 & 5.33 & 1.85 & 14 & 3.58 \\
\hline Muito severa & 5 & 12.5 & 7.6 & 3.16 & 15 & 4.30 \\
\hline \multirow{2}{*}{ Total } & 40 & 100 & 3.72 & 2.94 & 12.35 & 5.20 \\
\hline & $p=0.017$ & & \multicolumn{4}{|c|}{$r=0.91(p=0.03)$} \\
\hline
\end{tabular}

Legenda: №: Número de participantes. EVA Média: Valor médio de dor referida. QRM Médio: Pontuação média no QRM. Dp1: Desvio-padrão referente à EVA Média. Dp²: Desvio-padrão referente ao QRM Médio. r: Coeficiente de Correlação de Pearson. Fonte: Alves JCR, et al., 2021. 
Na medida de flexibilidade do tronco por meio do TSA a média de alcance foi de $23,19 \mathrm{~cm}( \pm 8,27)$. Na distribuição dos valores médios de alcance do TSA entre os níveis de incapacidade do QRM, o cálculo de correlação entre os níveis de incapacidade do QRM e o TSA médio resultou em um Coeficiente de Pearson de $-0,92(p=0,076)$ indicando alta correlação negativa, porém acima do nível de significância estatística (Tabela 3).

Tabela 3 - Valores de alcance médios no TSA distribuídos por níveis de incapacidade no QRM, $\mathrm{n}=40$.

\begin{tabular}{|c|c|c|c|c|c|c|}
\hline Incapacidade (QRM) & № & $\%$ & QRM Médio & $\mathrm{Dp}^{1}$ & TSA Médio & $\mathrm{Dp}^{2}$ \\
\hline Leve & 6 & 15 & 4 & 1.41 & 25.25 & 6.26 \\
\hline Moderada & 14 & 35 & 9.79 & 1.42 & 25.29 & 8.31 \\
\hline Grave & 15 & 37.5 & 15.53 & 2.07 & 21.27 & 8.77 \\
\hline Muito Grave & 5 & 12.5 & 20 & 0.71 & 20.6 & 8.96 \\
\hline \multirow{2}{*}{ Total } & 40 & 100 & 12.33 & 6.95 & 23.10 & 2.52 \\
\hline & $p=0.042$ & & \multicolumn{4}{|c|}{$r=-0.92(p=0.076)$} \\
\hline
\end{tabular}

Legenda: №: número de participantes. QRM Médio: Pontuação média no QRM. TSA Médio: Valor médio de alcance em cm no TSA. Dp': Desvio-padrão referente ao QRM Médio. Dp²: Desvio-padrão referente ao TSA Médio. r: Coeficiente de Correlação de Pearson. Fonte: Alves JCR, et al., 2021.

\section{DISCUSSÃO}

De acordo com os resultados do estudo, a idade média dos participantes foi de 46 anos e a maioria dos casos de lombalgia abordados foi em mulheres (62,5\%). Em um estudo de Romero DE, et al. (2018) realizado usando os dados do Instituto Brasileiro de Geografia e Estatística (IBGE) a prevalência do problema crônico de coluna foi de $18,5 \%$, sendo maior em mulheres e aumentando com a idade chegando a $28,1 \%$ aos 60 anos.

Em estudo de Bragada JE, et al. (2020), dois terços de uma amostra de 40 pacientes com diagnóstico de lombalgia foram constituídos por mulheres, sendo apontado pelos autores uma considerável documentação em diversos estudos da maior prevalência de lombalgia crônica nesta população. Tal achado pode ser devido a múltiplos fatores, como características fisiológicas (menor massa muscular e óssea e maior sobrecarga relacionada com gestações) ou a fatores psicológicos que levam a uma maior sensibilização à dor (MEUCCI RD, et al., 2015).

A ocupação mais frequente de "serviços domésticos" e o nível de escolaridade prevalente sendo o "ensino médio" aponta a alta incidência da lombalgia nesses grupos sociais. Segundo Zaina et al. (2020), o baixo nível socioeconômico com baixa renda e educação estão relacionados à lombalgia crônica incapacitante, devido, dentre outros fatores, a exposições ambientais, condições de trabalho, estilo de vida e menor disponibilidade de serviços de saúde.

O diagnóstico clínico mais prevalente observado foi a "lombalgia" e a queixa principal predominante foi a "dor lombar crônica". De acordo com Nijs J, et al. (2017), os preditores de dor e incapacidade persistente relacionada à lombalgia incluem fatores genéticos, estilo de vida, sociais e psicológicos e comorbidades, no entanto, nenhum fator de risco isolado é fortemente preditivo.

O nível de dor predominante medido pela EVA foi o de "dor moderada" com uma reduzida incidência nos níveis extremos da escala. Na algometria as maiores leituras de pressão necessárias ao início da queixa álgica foram nos participantes classificados no nível "sem dor" da EVA. Este achado demonstra que os casos classificados como "sem dor" no momento da avaliação apresentaram um maior limiar doloroso à pressão local com uma suposta correlação negativa entre as variáveis. Entretanto a análise estatística revelou uma baixa correlação entre "nível da EVA" e "algometria média".

Nos resultados da avaliação de incapacidade pelo QRM, a média do escore enquadrou-se no nível "moderado", porém observou-se que o nível de incapacidade "grave" foi o mais prevalente. Em estudo de Cargnin ZA, et al. (2019), envolvendo profissionais de enfermagem, a incapacidade funcional medida pelo questionário QRM foi baixa, com média de 7,52. Segundo o referido estudo a dor lombar crônica não parece prejudicar significativamente as atividades pessoais e o desempenho no trabalho nesta população específica. 
A lombalgia pode interferir na capacidade física do indivíduo e resultar em dependência para a realização das atividades de vida diária, como dificuldade para sentar, levantar e andar, o que afeta negativamente a qualidade de vida (SOUZA IMB, et al., 2019; BARBOSA AP, et al., 2015). Resultados de Nim CG, et al. (2021) demonstraram uma correlação positiva moderada entre rigidez da coluna lombar e Limiar de dor à pressão; maiores escores de rigidez foram associados a maiores limiares de dor mecânica profunda.

O presente estudo apresentou associação do nível de dor "muito severa" da EVA com médias de pontuações mais elevadas do QRM, com alta correlação positiva entre as variáveis. Em um estudo comparativo, a intensidade da dor apresentou correlação moderada e significativa com a incapacidade, de maneira que quanto mais intensa a dor, maior a incapacidade do indivíduo avaliado (RIBEIRO RP, et al., 2018).

A média de alcance no TSA referente à flexibilidade do tronco foi de $23,19 \mathrm{~cm}$ representando um resultado comprável ao estudo de Lima BA, et al. (2020) que obteve média de $29 \mathrm{~cm}$ de alcance, no qual os autores ponderam que em indivíduos com lombalgia é observado que a mobilidade se encontra reduzida se comparada com indivíduos saudáveis.

Na distribuição dos resultados do TSA entre os níveis de incapacidade do QRM, os menores alcances obtidos foram no nível de incapacidade "muito grave" com alta correlação negativa entre as variáveis. Este achado pressupõe que quanto maior o nível de incapacidade, menor poderá ser a mobilidade e flexibilidade do tronco, porém a correlação observada não foi significativa estatisticamente.

Em estudo de Ribeiro RP, et al. (2018), os indivíduos com dor lombar crônica não apresentaram comprometimento da flexibilidade da coluna vertebral quando comparados com indivíduos sem dor. Entretanto, testes de correlação mostraram que quanto maior a intensidade da dor, maior é a incapacidade e menores são os resultados no banco de Wells.

Um dos fatores associados à dor na região lombar é o déficit de flexibilidade do tronco e membros inferiores. O encurtamento dos músculos isquiotibiais pode girar a pelve posteriormente reduzindo a curvatura lombar, alterando o ritmo lombopélvico, o que gera movimentos compensatórios e dor. Indivíduos nesta condição apresentam diversas alterações funcionais, dentre elas a perda de estabilidade, diminuindo a funcionalidade da coluna (TOZIM BM, et at., 2020).

Merino GSAD, et al. (2018) enfatizam a importância de considerar o projeto centrado no usuário no desenvolvimento de novos produtos, como forma de garantir o atendimento das necessidades funcionais do usuário e o cumprimento do objetivo do produto. Na concepção de produtos de saúde, notadamente de recursos terapêuticos, os achados clínicos e funcionais do público-alvo devem ser levados em consideração na definição de requisitos e atributos do produto. Os resultados do estudo fornecem indícios de que um recurso terapêutico voltado a pacientes com dor lombar deverá apresentar como requisitos principais a capacidade de reduzir a dor e promover o ganho de mobilidade do tronco, levando a uma consequente melhora da capacidade funcional.

As limitações do estudo relacionam-se principalmente a baixa amplitude da amostra, devida, entre outros fatores, à dificuldade dos participantes em engajar-se voluntariamente em estudos de pesquisas no atual cenário de pandemia. Não obstante, foi possível observar resultados potencialmente úteis ao estudo dos processos envolvidos na manifestação dos sintomas e nas repercussões funcionais da dor lombar e no aprimoramento dos meios de tratamento desta condição, especificamente ao embasar o desenvolvimento de um recurso terapêutico inovador a ser abordado em publicação futura.

\section{CONCLUSÃO}

O delineamento do perfil algométrico e funcional dos pacientes com dor lombar avaliados no estudo demonstrou que a maioria dos participantes foi de mulheres, com idade média de 46 anos. O nível de dor moderada foi predominante na avaliação pela EVA com baixa correlação negativa com a algometria digital. O nível de incapacidade grave foi o mais prevalente na avaliação pelo QRM, com alta correlação positiva 
com a intensidade de dor da EVA e alta correlação negativa com o alcance no TSA. Os presentes achados indicam a necessidade de que o recurso terapêutico a ser projetado, apresente atributos de funcionalidade focados na redução da dor, na melhora da mobilidade e na capacidade funcional dos usuários. Perspectivas de estudos futuros neste contexto incluem investigações com amostras mais extensas e abrangentes, e que considerem os efeitos dos recursos terapêuticos na condição clínica dos pacientes.

\section{FINANCIAMENTO}

Estudo executado com recursos provenientes da Fundação Amazônia de Amparo a Estudos e Pesquisas (FAPESPA) por meio da CHAMADA No 012/2017.

\section{REFERÊNCIAS}

1. BARBOSA AP, et al. Nível de atividade física e qualidade de vida: um estudo comparativo entre idosos dos espaços rural e urbano. Revista Brasileira de Geriatria e Gerontologia, 2015; 18(4): 743-754.

2. BERNARDINO SN. Medidas dos limiares dolorosos por meio de algometria de pressão em pacientes com cefaleia primária. Dissertação de Mestrado. Universidade Federal de Pernambuco, 2012; 57p.

3. BRAGADA JE, et al. O Impacto da Dor Lombar Crónica na Funcionalidade. Revista da SPMFR, 2020; 32(3): 95-100.

4. CARGNIN ZA, et al. Functional disability and pain intensity in nonspecific chronic low back pain in nursing workers. Cogitare enferm. [Internet], 2019; 32(6): 707-13.

5. CARVALHO AS. Sistema de avaliação e tratamento da coluna lombar com o uso de tração mecânica. Tese (Doutorado) - Universidade Estadual Paulista, Faculdade de Engenharia, 2015; 72p.

6. CHETTY L. A Critical Review of Low Back Pain Guidelines. Workplace Health \& Safety, 2017; 65(9): 388-394.

7. $\mathrm{CHOl} \mathrm{J}$, et al. Influences of spinal decompression therapy and general traction therapy on the pain, disability, and straight leg raising of patients with intervertebral disc herniation. J. Phys. Ther. Sci, 2015; 27: 481-483.

8. GRANDE-ALONSO M, et al. A. Relação entre busca de assistência e expansão da dor em pacientes com lombalgia crônica inespecífica. PeerJ, 2020; 8: e8756.

9. GRAVEN-NIELSEN T, ARENDT-NIELSEN L. Assessment of mechanisms in localized and widespread musculoskeletal pain. Nat Rev Rheumatol, 2010; 6: 599-606.

10. HARTVIGSEN J, et al. What low back pain is and why we need to pay attention. Lancet, 2018; 391(10137): 23562367.

11. LIMA BA, et al. Correlação entre limiar de tolerância de dor à pressão, força e mobilidade lombar em universitárias. Revista Saúde (Sta. Maria), 2020; 46(1): 1-9.

12. MARTINEZ JE, et al. Análise da aplicabilidade de três instrumentos de avaliação de dor em distintas unidades de atendimento: ambulatório, enfermaria e urgência. Rev Bras Reumatol, 2011; 51 (4): 299-308.

13. MARTINS ABQP, FIGUEIREDO SP. Banco de Wells Como Indicador no Tratamento Fisioterápico. Anais do Congresso Brasileiro de Fisioterapia, 2016; 1(1).

14. MENDONÇA AG. Dor lombar em hospitais financiados pelo Sistema Único de Saúde brasileiro entre 2013 e 2018 : custos diretos, prevalência e perfil dos pacientes notificados. Dissertação (Mestrado em Reabilitação e Desempenho Funcional) - Programa de Pós-Graduação em Reabilitação e Desempenho Funcional, Universidade Federal dos Vales do Jequitinhonha e Mucuri, Diamantina, 2020; 76p.

15. MERINO GSAD, et al. Contribuições do Design na promoção da autonomia em um Hospital Psiquiátrico de Santa Catarina. 13ํㅡㄹ Congresso Brasileiro de Pesquisa e Desenvolvimento em Design, Joinville (SC), 2018.

16. MEUCCI RD, et al. Prevalence of chronic low back pain: systematic review. Rev Saúde Pública, 2015; $49: 73$.

17. MONTEIRO J, et al. Questionário de incapacidade de Roland Morris: adaptação e validação para a população portuguesa com lombalgia. Acta Médica Portuguesa, 2010; 23: 761-766.

18. NIJS J, et al. Sleep disturbances and severe stress as glial activators: key targets for treating central sensitization in chronic pain patients? Expert Opinion on Therapeutic Targets, 2021; 8: 817-826.

19. NIM CG, et al. A cross-sectional analysis of persistent low back pain, using correlations between lumbar stiffness, pressure pain threshold, and heat pain threshold. Chiropr Man Therap, 2021;29: 34.

20. POLLI GR, et al. Atividade física e dor lombar em brasileiros: uma revisão sistemática. Rev. Bras. Ativ. Fís. Saúde, 2018; 23: e0047.

21. RAMOS M, et al. Effects of neural mobilization on individuals with chronic low back pain. BrJP, 2020; 3(3): $205-212$.

22. RIBEIRO RP, et al. Relação entre a dor lombar crônica não específica com a incapacidade, a postura estática e a flexibilidade. Fisioterapia e Pesquisa [online], 2018; 25(4): 425-431.

23. ROMERO DE, et al. Prevalência, fatores associados e limitações relacionados ao problema crônico de coluna entre adultos e idosos no Brasil. Cadernos de saúde pública, 2018; 34: e00012817.

24. SCHIMIDT GJ, et al. Comparative evaluation of the psychometric properties of orthopedic scales for low back pain. Coluna/Columna, 2019; 18(4): 308-312.

25. SIVIERO D, et al. Confiabilidade inter-avaliador para determinação do limiar de dor pelo dolorímetro de pressão. FIEP BULLETIN, 2013; 83.

26. SOUZA IMB, et al. Prevalence of low back pain in the elderly population: a systematic review. Clinics, [Online], 2019; 74: e789.

27. TOZIM BM, et al. Reclutamiento y co-contracción muscular durante la marcha en mujeres jóvenes con dolor lombar crónica. Fisioter. mov, 2020; 33.

28. ZAINA F, et al. Low back pain rehabilitation in 2020: new frontiers and old limits of our understanding. Eur J Phys Rehabil Med, 2020; 56: 212-9. 